\title{
Cone beam computed tomography-guided thin/ultrathin bronchoscopy for diagnosis of peripheral lung nodules: a prospective pilot study
}

\author{
Roberto F. Casal ${ }^{1}$, Mona Sarkiss ${ }^{2}$, Aaron K. Jones ${ }^{3}$, John Stewart ${ }^{4}$, Alda Tam ${ }^{5}$, Horiana B. Grosu ${ }^{1}$, David E. Ost ${ }^{1}$, \\ Carlos A. Jimenez ${ }^{1}$, George A. Eapen ${ }^{1}$ \\ ${ }^{1}$ Department of Pulmonary Medicine, ${ }^{2}$ Department of Anesthesiology and Preoperative Medicine, ${ }^{3}$ Department of Imaging Physics, ${ }^{4}$ Department of \\ Pathology, ${ }^{5}$ Department of Interventional Radiology, The University of Texas MD Anderson Cancer Center, Houston, Texas, USA \\ Contributions: (I) Conception and design: All authors; (II) Administrative support: RF Casal; (III) Provision of study materials or patients: RF Casal; \\ (IV) Collection and assembly of data: RF Casal, AK Jones; (V) Data analysis and interpretation: All authors; (VI) Manuscript writing: All authors; (VII) \\ Final approval of manuscript: All authors. \\ Correspondence to: Roberto F. Casal, MD. Associate Professor of Medicine. Department of Pulmonary Medicine, The University of Texas MD \\ Anderson Cancer Center, 1400 Pressler Street, Houston, Texas 77030, USA. Email: rfcasal@mdanderson.org.
}

Background: Despite advances in bronchoscopy, its diagnostic yield for peripheral lung lesions continues to be suboptimal. Cone beam computed tomography (CBCT) could be utilized to corroborate the accuracy of our bronchoscopic navigation and hopefully increase its diagnostic yield. However, data on radiation exposure and feasibility of CBCT-guided bronchoscopy is scarce.

Methods: Prospective pilot study of bronchoscopy for peripheral lung nodules under general anesthesia with thin/ultrathin bronchoscope, radial-probe endobronchial ultrasound (RP-EBUS), and CBCT. Main objective was to estimate radiation dose and secondary objective was the additional value of CBCT in terms of navigational and diagnostic yield.

Results: A total of 20 patients were enrolled. Median lesion size was 2.1 (range, 1.1-3) cm and distance from pleura was 2.1 (range, $0-2.8) \mathrm{cm}$. "Bronchus sign" was present in $12(60 \%)$ of the lesions. Totally, 12 lesions (60\%) were invisible on fluoroscopy. CBCT identified atelectasis obscuring the target in 4 cases (20\%). Eleven patients (55\%) underwent 1 CBCT scan and 9 patients (45\%) 2 . The mean estimated effective dose (E) to patients resulting from CBCT ranged between 8.6 and $23 \mathrm{mSv}$, depending on utilized conversion factors. Both pre-CBCT navigation and diagnostic yield were 50\%. Additional post-CBCT maneuvers increased navigation yield to $75 \%(\mathrm{P}=0.02)$ and diagnostic yield to $70 \%(\mathrm{P}=0.04)$. One patient developed a pneumothorax.

Conclusions: CBCT-guided bronchoscopy is associated with an acceptable radiation dose. CBCT may potentially increase both navigation and diagnostic yield of thin/ultrathin bronchoscopy for peripheral lung nodules. The above findings as well as the incidental but relevant finding of intra-procedural atelectasis need to be confirmed in larger prospective studies.

Trial registration: This study is registered in ClinicalTrials.gov as number NCT02978170.

Keywords: Guided-bronchoscopy; cone beam computed tomography (CBCT); ultrathin bronchoscopy

Submitted Jun 26, 2018. Accepted for publication Oct 07, 2018.

doi: $10.21037 /$ jtd.2018.11.21

View this article at: http://dx.doi.org/10.21037/jtd.2018.11.21 


\section{Introduction}

Diagnosis of peripheral lung nodules can be achieved with a variety of techniques, including bronchoscopy, computed tomography (CT)-guided needle biopsy, and video-assisted thoracoscopic surgery (1). Despite recent advances in bronchoscopy, its diagnostic yield continues to be suboptimal and varies substantially among different publications, ranging in some of them from $38 \%$ to $74 \%(2-5)$.

Conventional multidetector computed tomography in combination with bronchoscopy with a standardsize bronchoscope has not been proven to be superior to the above described techniques of guided-bronchoscopy (6-8). On the other hand, a single center retrospective study showed the combination of standard CT-guidance with ultrathin bronchoscopes to have a diagnostic yield of almost $80 \%(9)$.

Cone beam computed tomography (CBCT) is a newer modality that has been widely adopted by interventional radiologists (10). The system is compact enough to mount on a moving $\mathrm{C}$-arm, thereby allowing the patient to remain stationary during the examination. In a single orbit around the patient, a complete volumetric dataset covering a large anatomic region of interest is generated, and from this a submillimeter isotropic reconstruction can be created. Data on the use of this technology in combination with bronchoscopy is very scant (11-14). Moreover, the combination of CBCT with thin or ultrathin scopes has not yet been reported.

We hypothesized that CBCT-guided thin/ultrathin bronchoscopy would be safe in terms of radiation exposure and would improve navigational and diagnostic yield for peripheral lung nodules suspicious of malignancy.

\section{Methods}

\section{Study setting and subjects}

The study was performed at the University of Texas MD Anderson Cancer Center and it was approved by its Institutional Review Board (protocol 2016-0466). All participants gave informed consent before taking part in the study. Adult patients referred to our pulmonary department for diagnosis of lung nodules of $1-3 \mathrm{~cm}$ located in the outer two thirds of the lung were included in this trial. Patients with suspected lymph node metastases [lymph nodes enlarged by CT or with standardized uptake values (SUV) of $>2.5$ on positron emission tomography (PET)-
CT], pregnant patients, and patients with contraindication for general anesthesia were excluded. Enrollment occurred between February 2017 and February 2018.

\section{Study design}

This was a prospective observational cohort study in which patients underwent guided-bronchoscopy for peripheral lung nodules with the combination of thin/ultrathin bronchoscope and radial-probe endobronchial ultrasound (RP-EBUS), and with the addition of CBCT to confirm location of our EBUS probe or sampling instruments with respect to our targets, and to help navigate and sample targets. The main objective was to describe radiation dose associated with CBCT. Secondary objectives included the description of the additional value of CBCT in terms of navigational and diagnostic yield. "Navigational yield" was defined as the proportion of patients in whom navigation was successful (the lesion was identified as a positive or inconclusive image by RP-EBUS and CBCT confirmed that RP-EBUS was in contact with the lesion). The presence of lung parenchyma in between radial probe and target was considered as lack of contact. RP-EBUS images were categorized as "positive" (image of suspected target, either concentric or eccentric), "negative" ("snow storm" or aerated lung), or "inconclusive" (image is abnormal but it does not match any of the prior 2 definitions). Cases where a positive or inconclusive RP-EBUS image was obtained and CBCT proved that the RP-EBUS probe was not in contact with the target were classified as navigation failure. "Diagnostic yield" was defined as the proportion of patients in whom diagnostic samples were obtained. We defined "diagnostic samples" as those that identified a malignant or benign process. Benign pathology was either confirmed surgically or clinically and radiographically (6-month follow-up). Samples with bronchial cells, blood, or alveolar macrophages were considered "non-diagnostic" even if the lesion resolved during the follow-up period. Samples with non-specific inflammation were considered diagnostic only if they were confirmed by surgical pathology or they resolved or improved during follow-up period. The "postCBCT yield" was defined as the proportion of patients in whom CBCT-imaging allowed the operator to reach the lesion that was not reached prior to CBCT ("post-CBCT navigational yield") or to obtain a diagnosis that was not obtained prior to CBCT ("post-CBCT diagnostic yield") (Figure 1). Total bronchoscopy time was measured in minutes from "first scope in" until "last scope out". 


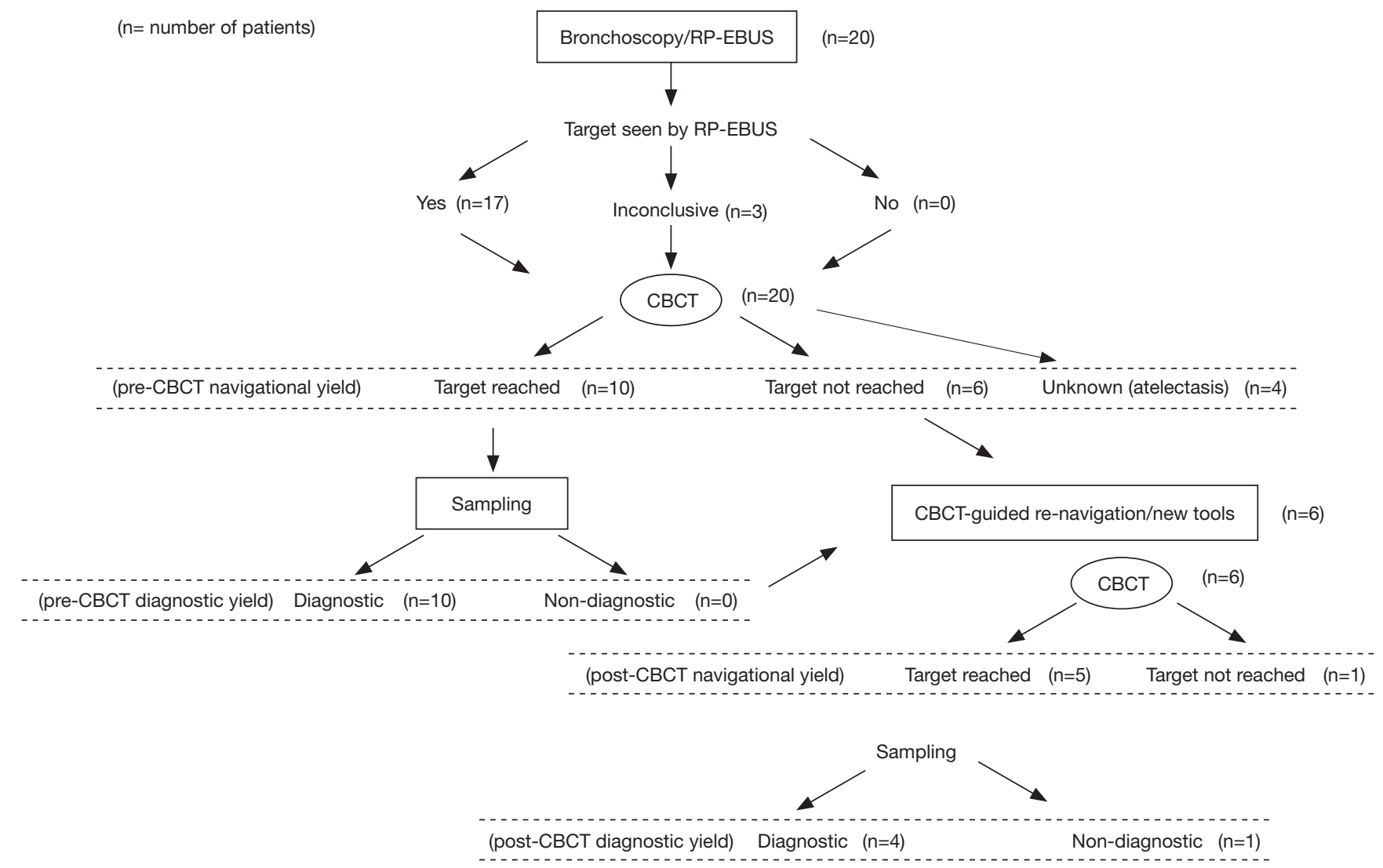

Figure 1 Study flowchart. RP-EBUS, radial-probe endobronchial ultrasound; CBCT, cone beam computed tomography.

\section{Study interventions}

The flow of the study procedures is depicted in Figure 1. Bronchoscopic navigation was planned based on the analysis of pre-procedural chest CT scans with available axial, sagittal and coronal cuts. All bronchoscopies were performed under general anesthesia via laryngeal mask airway and rapid-on site cytology evaluation (ROSE) was available in all cases. Initial navigation was performed with a thin scope with an outer diameter of $4.2 \mathrm{~mm}$ and a working channel of 2 mm (Olympus BF-P190, Olympus America Inc., Cypress, USA). RP-EBUS (UM-S20-17S, Olympus America Inc., Cypress, USA) and fluoroscopy were utilized in all cases. The operator would navigate to the best of his ability to reach the target (identify target with RPEBUS) and this would then be corroborated with CBCT to determine navigation success (Figure 2). If navigation was deemed successful, then samples were taken. If navigation was not successful (Figure 3) or samples were non-diagnostic on ROSE, then the operator-based on CBCT image findings-would either re-navigate to the lesion, change angle/orientation of scope, change the scope (utilizing an ultrathin scope Olympus BF-XP190, Olympus America Inc.), or use new sampling tools. The success of these new maneuvers (re-navigation, change of scope or scope angle, or use of different tools) conformed the "post-CBCT navigational and diagnostic yield". A second CBCT was performed to confirm additional navigational yield when renavigation was performed after the initial CBCT. Sampling was performed with a combination of transbronchial needle aspiration (TBNA), cytology brushing, and forceps biopsy. When an infectious diagnosis was suspected on ROSE, a bronchoalveolar lavage (BAL) was performed.

A single Siemens Artis dTA angiography system (Siemens Healthineers, Malvern, PA, USA) with a $40 \times 30 \mathrm{~cm}$ detector was used for all procedures in the study. The CBCT imaging protocol used (DynaCT) was characterized by the following parameters: $8 \mathrm{~s}$ rotation time, $200^{\circ}$ gantry rotation, $0.5 \%$ projection, 396 total projections, and a detector dose of $0.36 \mu \mathrm{Gy} /$ frame. The patient's arms were 

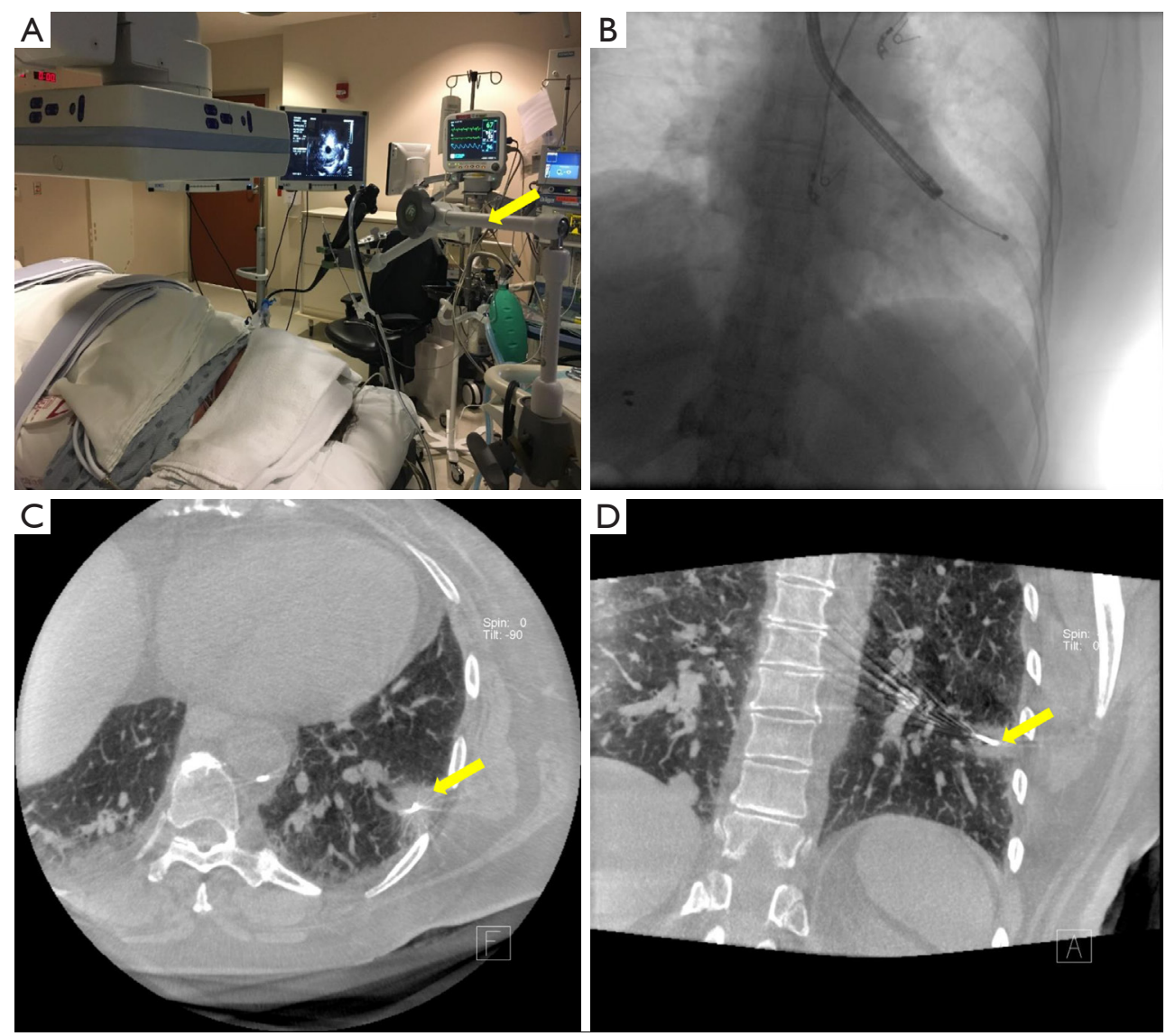

Figure 2 CBCT-guided bronchoscopy case illustration. (A) Dedicated mechanical arm attached to bronchoscopy cart and holding the bronchoscope in place during scanning (arrow); (B) fluoroscopy image showing guide-sheath proximal to left lower lobe lesion (lesion is not visible by fluoroscopy); (C,D) show axial and coronal cuts of CBCT showing tip of RP-EBUS within the target. CBCT, cone beam computed tomography; RP-EBUS, radial-probe endobronchial ultrasound.

raised above his/her head, a "breath-hold" maneuver was performed by the anesthesiologists, and the CBCT scan was then performed. A dedicated arm attached to the bronchoscopy cart (The Arm, Neuwave Medical Inc., WI, USA) was utilized to hold the bronchoscope in position so that the operators could leave the room during CBCT scan (Figure 2). Radiation dose metrics from fluoroscopy and CBCT, reference air kerma (Kar) and kerma area product $\left(\mathrm{P}_{\mathrm{KA}}\right)$ were obtained.

\section{Statistics}

Basic descriptive statistics were utilized for patient and procedures description. Categorical data was analyzed with Fisher exact test. McNemar's Test was utilized to determine the difference in navigational and diagnostic yield before and after CBCT (Stata/SE 14.1, College Station, TX, USA).

\section{Study registration}

This study is registered in ClinicalTrials.gov as number NCT02978170.

\section{Results}

A total of 32 patients were screened. Two patients refused, and 10 patients met an exclusion criterion. Twenty patients were enrolled in the study. Baseline characteristics are depicted in Table 1. Fifteen patients were female (75\%), 13 patients were prior or current smokers (65\%), and 9 patients had a prior baseline malignancy (45\%). Median lesion 

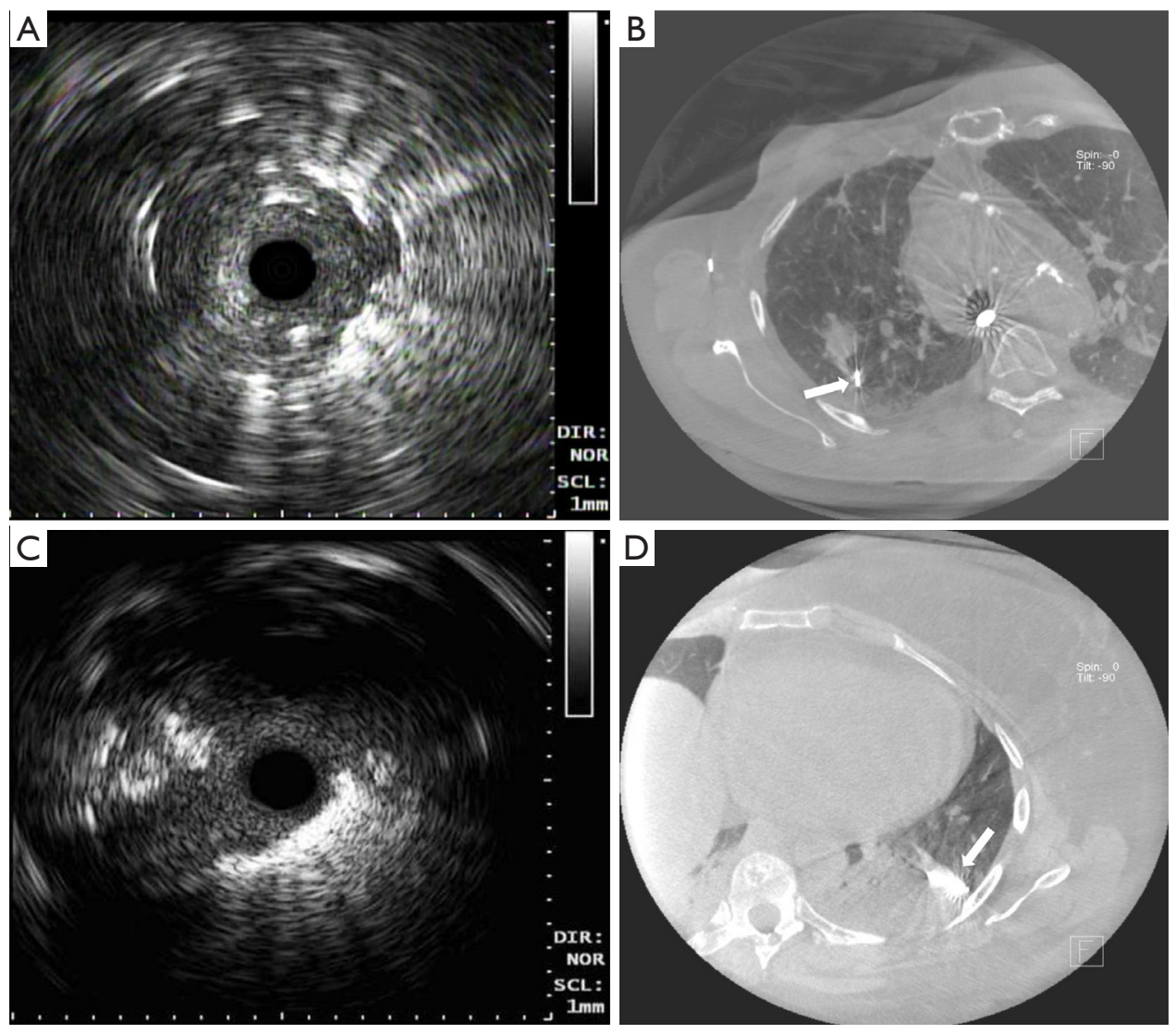

Figure 3 CBCT demonstrating unsuccessful navigation and atelectasis obscuring target. (A) RP-EBUS showing a falsely "positive" image finding; (B) CBCT correlation of image "A", showing that the RP-EBUS is not in contact with the target (unsuccessful navigation); (C) RPEBUS showing a "positive" image finding; (D) CBCT correlation of image "C", showing the RP-EBUS surrounded by atelectasis which are obscuring the target. CBCT, cone beam computed tomography; RP-EBUS, radial-probe endobronchial ultrasound.

size was 2.1 (range, 1.1-3) cm and median distance from pleura was 2.1 (range, $0-2.8) \mathrm{cm}$. Lesion density by CT was classified as "solid" in 13 cases $(65 \%)$, and "bronchus sign" was present in $12(60 \%)$ of the 20 lesions. Bronchoscopic technical aspects are described in Table 2. Median total bronchoscopy time was 62.5 (range, 49-96) minutes. Median peripheral bronchoscopy time was 50 (range, 30-76) minutes. EBUS staging was performed after peripheral bronchoscopy in 12 cases $(60 \%)$ with a median of 3.5 (range, 1-7) lymph nodes per patient being sampled. Totally, 12 targets $(60 \%)$ were not visible on fluoroscopy. All 8 targets that were fluoroscopy visible were seen by RP-EBUS (pre-CBCT), and 6 of them were confirmed by CBCT. Of note, atelectasis in dependent areas was demonstrated in 8 patients (40\%), and in 4 cases atelectasis was actually obscuring the target. None of the atelectasis that developed was visible with fluoroscopy; it was only visible by CBCT. Eleven patients (55\%) received 1 CBCT scan, and $9(45 \%)$ received 2 CBCT scans. Six patients underwent a second CT scan due to initial unsuccessful navigation and after new bronchoscopic maneuvers (of note, 5 of these 6 were fluoroscopy invisible), and 3 patients had their second CBCT done after recruitment maneuvers in an attempt to resolve atelectasis. Both temporary increase of PEEP and tidal volumes failed to resolve atelectasis. Radiation dose indices were available in 17 cases (Table 3). Using the range of conversion factors reported previously in the literature, 0.17 (15) to $0.45 \mathrm{mSv} / \mathrm{Gy}-\mathrm{cm}^{2}$ (16) the mean effective dose (E) to patients from CBCT alone was estimated to range between 8.6 to $23 \mathrm{mSv}$, and the total procedural $\mathrm{E}$ (accounting for fluoroscopy as well) ranged from 11 to $29 \mathrm{mSv}$. CBCT-guided bronchoscopy data, navigation and diagnostic yields, and final diagnoses are summarized in 
Table 1 Patient and target characteristics

\begin{tabular}{|c|c|}
\hline Baseline patient and target characteristics & Values \\
\hline Age, median [range] & $70[48-86]$ \\
\hline \multicolumn{2}{|l|}{ Gender, n [\%] } \\
\hline Male & $5[25]$ \\
\hline Female & $15[75]$ \\
\hline \multicolumn{2}{|l|}{ Smoking history, $\mathrm{n}[\%]$} \\
\hline Never smoker & $7[35]$ \\
\hline Prior smoker & $11[55]$ \\
\hline Current smoker & $2[10]$ \\
\hline \multicolumn{2}{|l|}{ ASA score, $\mathrm{n}[\%]$} \\
\hline II & $6[30]$ \\
\hline III & $14[70]$ \\
\hline \multicolumn{2}{|l|}{ Baseline malignancy } \\
\hline Lung & 2 \\
\hline Breast & 3 \\
\hline Thyroid & 2 \\
\hline Colorectal & 1 \\
\hline Others & 1 \\
\hline Target size $(\mathrm{cm})$, median [range] & $2.1(1.1-3)$ \\
\hline Distance to pleura $(\mathrm{cm})$, median [range] & $2.1(0-2.8)$ \\
\hline \multicolumn{2}{|l|}{ Computed tomography density, n [\%] } \\
\hline Solid & 13 [65] \\
\hline Semi-solid & $6[30]$ \\
\hline Ground-glass & $1[5]$ \\
\hline Positive bronchus sign, $n$ [\%] & $12[60]$ \\
\hline \multicolumn{2}{|l|}{ FDG-avidity by PET, n [\%] } \\
\hline Avid & $14[70]$ \\
\hline Not avid & $1[5]$ \\
\hline Not available & 5 [25] \\
\hline \multicolumn{2}{|l|}{ Target location, n [\%] } \\
\hline RUL & $7[35]$ \\
\hline RML & $4[20]$ \\
\hline RLL & $1[5]$ \\
\hline LUL & $5[25]$ \\
\hline LLL & $3[15]$ \\
\hline
\end{tabular}

ASA, American Society of Anesthesia; FDG, fluorodeoxyglucose; $\mathrm{PET}$, positron emission computed tomography; RUL, right upper lobe; RML, right middle lobe; RLL, right lower lobe; LUL, left upper lobe; LLL, left lower lobe.
Table 2 Bronchoscopic technical data

\begin{tabular}{|c|c|}
\hline Bronchoscopy technical data & Values \\
\hline \multicolumn{2}{|l|}{ Bronchoscopy time (min), median [range] } \\
\hline Total & $62.5[49-96]$ \\
\hline Peripheral bronchoscopy & $50[30-76]$ \\
\hline EBUS staging & 17 [10-29] \\
\hline Fluoroscopy time (min), median [range] & $8.6(5.0-15.4)$ \\
\hline \multicolumn{2}{|l|}{ CBCT scan, n [\%] } \\
\hline One & $11[55]$ \\
\hline Two & 9 [45] \\
\hline \multicolumn{2}{|l|}{ Target visible with fluoroscopy, n [\%] } \\
\hline Yes & $8[40]$ \\
\hline No & 12 [60] \\
\hline \multicolumn{2}{|l|}{ Bronchoscope, n [\%] } \\
\hline Thin & $15[75]$ \\
\hline Ultrathin & $5[25]$ \\
\hline \multicolumn{2}{|l|}{ EBUS staging } \\
\hline Performed, n [\%] & 12 [60] \\
\hline LN sampled, median [range] & $3.5[1-7]$ \\
\hline \multicolumn{2}{|l|}{$\begin{array}{l}\text { Bronchoscopic tools/procedures, } \\
\text { median [range] }\end{array}$} \\
\hline Guide-sheath & $13[65]$ \\
\hline Edge catheter & $1[5]$ \\
\hline TBNA & $17[85]$ \\
\hline Cytology brush & 9 [45] \\
\hline Forceps biopsy & 13 [65] \\
\hline Triple-needle-brush & 5 [25] \\
\hline BAL & $6[30]$ \\
\hline \multicolumn{2}{|l|}{$\begin{array}{l}\text { Number of samples per patient, median } \\
\text { [range] }\end{array}$} \\
\hline TBNA & $5[3-7]$ \\
\hline Cytology brush & $2[1-3]$ \\
\hline Forceps biopsy & $4[3-5]$ \\
\hline Triple-needle-brush & 2 \\
\hline
\end{tabular}

EBUS, endobronchial ultrasound; CBCT, cone-beam computed tomography; LN, lymph node; TBNA, transbronchial-needle aspiration; BAL, bronchoalveolar lavage. 
Table 3 Radiation dose

\begin{tabular}{lccc}
\hline Radiation data & Mean & Min & Max \\
\hline Number of CBCT & 1.5 & 1 & 2 \\
$\mathrm{P}_{\mathrm{KA}}$ from CBCT $\left(\mathrm{Gy}-\mathrm{cm}^{2}\right)^{*}$ & 50.45 & 5.43 & 114.89 \\
$\begin{array}{l}\text { Total } \mathrm{P}_{\mathrm{KA}}\left(\mathrm{Gy}-\mathrm{cm}^{2}\right)^{*} \text { for the } \\
\text { entire procedure }\end{array}$ & 64.57 & 6.14 & 66.75 \\
$\begin{array}{l}\text { Total fluoroscopy time } \\
\text { (minutes) } \\
\text { procedure }\end{array}$ & 8.6 & 5.0 & 15.4 \\
\hline
\end{tabular}

*, radiation dose indices, including fluoroscopy time, are characterized by a lognormal distribution, and the arithmetic mean of this distribution is reported here. $\mathrm{P}_{\mathrm{KA}}$, kerma area product; $\mathrm{CBCT}$, cone-beam computed tomography.

Table 4. Both pre-CBCT navigation and diagnostic yields were $50 \%$ (10 out of 20 patients). With additional postCBCT maneuvers performed in 10 remaining patients, the post-CBCT navigation yield was increased to $75 \%(\mathrm{P}=0.02)$ and the post-CBCT diagnostic yield was increased to $70 \%$ $(\mathrm{P}=0.04)$. The 4 additional diagnoses obtained post- CBCT included 3 cases of adenocarcinoma of the lung and 1 case of Mycobacterium avium complex (MAC) infection. Target characteristics of these four additional cases diagnosed post-CBCT are depicted in Table 5. These 4 targets were small in comparison with the rest of the cases $[1.45 \pm 0.45 \mathrm{vs}$. $2.25 \pm 0.40 \mathrm{~cm}$, mean \pm standard deviation $(\mathrm{SD}), \mathrm{P}=0.003]$.

The only complication that occurred during our trial was 1 case of pneumothorax which required chest-tube insertion. The pneumothorax was not present during CBCT acquisition or post-procedure chest $\mathrm{X}$-ray, but it was found 24 hours later when patient presented with mild chest pain and dyspnea.

\section{Discussion}

The results of our study suggest that CBCT-guided bronchoscopy is associated with acceptable radiation dose, and that the use of CBCT may potentially increase both navigation and diagnostic yield of thin/ultrathin bronchoscopy for peripheral lung nodules. Bronchoscopic maneuvers performed after reviewing initial CBCT resulted in a $25 \%$ absolute increase in navigation yield and $20 \%$ absolute increase in diagnostic yield. To the best of our knowledge, this is the first prospective study evaluating the additional value of CBCT in peripheral bronchoscopy with the report of results obtained before and after analyzing
Table 4 CBCT-guided bronchoscopy findings, navigational and diagnostic yield

\begin{tabular}{|c|c|}
\hline CBCT-guided bronchoscopy findings & Values, n (\%) \\
\hline \multicolumn{2}{|l|}{ RP-EBUS images } \\
\hline Eccentric & $11[55]$ \\
\hline Concentric & $6[30]$ \\
\hline Inconclusive & $3[15]$ \\
\hline \multicolumn{2}{|l|}{ RP-EBUS position per $1^{\text {st }} \mathrm{CBCT}$} \\
\hline Contact with lesion & $10[50]$ \\
\hline Central & $5[25]$ \\
\hline Peripheral & $5[25]$ \\
\hline \multicolumn{2}{|l|}{ Atelectasis } \\
\hline Any & $8[40]$ \\
\hline Obscuring target & $4[20]$ \\
\hline \multicolumn{2}{|l|}{ Pre-СВСТ navigational yield } \\
\hline Successful navigation & $10[50]$ \\
\hline Unsuccessful navigation & $6[30]$ \\
\hline Unknown (atelectasis) & $4[20]$ \\
\hline \multicolumn{2}{|l|}{ Post-CBCT maneuvers } \\
\hline Re-navigation & $6[30]$ \\
\hline Change of scope orientation & $3[15]$ \\
\hline New tool/s & $1[5]$ \\
\hline \multicolumn{2}{|l|}{ Post-CBCT navigational yield } \\
\hline Successful navigation & $15[75]^{*}$ \\
\hline Unsuccessful navigation & $1[5]$ \\
\hline Unknown (atelectasis) & $4[20]$ \\
\hline \multicolumn{2}{|l|}{ Diagnostic yield } \\
\hline Pre-CBCT & $10[50]^{*}$ \\
\hline Post-CBCT & $14[70]$ \\
\hline \multicolumn{2}{|l|}{ Bronchoscopic diagnosis } \\
\hline Adenocarcinoma of the lung & $8[40]$ \\
\hline Squamous cell carcinoma of the lung & $3[15]$ \\
\hline$M A C$ & $1[5]$ \\
\hline Necrotizing pneumonia (MRSA) & $1[5]$ \\
\hline Chronic inflammation & $1[5]$ \\
\hline
\end{tabular}

₹, $\mathrm{P}=0.02 ;{ }^{*}, \mathrm{P}=0.04$. CBCT, cone-beam computed tomography; RP-EBUS, radial-probe endobronchial ultrasound; MRSA, methicillin-resistant staphylococcus aureus; MAC, Mycobacterium avium complex. 
Table 5 Post CBCT additional diagnoses

\begin{tabular}{|c|c|c|c|c|c|c|c|c|}
\hline $\begin{array}{l}\text { Post-CBCT } \\
\text { diagnoses }\end{array}$ & $\begin{array}{l}\text { Target size } \\
\quad(\mathrm{cm})\end{array}$ & $\begin{array}{l}\text { Distance to } \\
\text { pleura }(\mathrm{cm})\end{array}$ & CT density & $\begin{array}{l}\text { "Bronchus } \\
\text { sign" }\end{array}$ & $\begin{array}{c}\text { Fluoroscopy } \\
\text { visible }\end{array}$ & Location & RP-EBUS image & $\begin{array}{c}\text { Post-CBCT } \\
\text { maneuver }\end{array}$ \\
\hline Adenocarcinoma & 1.4 & 2.8 & Solid & No & No & RUL & Eccentric & Change of tool angle \\
\hline Adenocarcinoma & 1.1 & 1.5 & Semi-solid & Yes & No & RUL & Inconclusive & Re-navigation \\
\hline
\end{tabular}

CBCT, cone-beam computed tomography; RP-EBUS, radial-probe endobronchial ultrasound; RUL, right upper lobe; LUL, left upper lobe; LLL, left lower lobe; MAC, Mycobacterium avium complex.

CBCT images. An unexpected, yet very interesting finding, was the detection by CBCT of atelectasis that was not visible with fluoroscopy, and the fact that in 4 cases atelectasis obscured the target.

The relatively wide range of mean $\mathrm{E}$ to patients reported in our study-ranging from 11 to $29 \mathrm{mSv}$ for the total procedure including fluoroscopy and CBCT-is not more than an estimation based on the lowest and highest conversion factors previously reported $(15,16)$, and, if the conversion factors previously reported for the same model of fluoroscope are used (15), the E resulting from this study is at the low end of the reported range. Calculating $\mathrm{E}$ from the $\mathrm{P}_{\mathrm{KA}}$ reported by fluoroscope requires that the irradiation geometry is known. However, the irradiation geometry during CBCT-guided transbronchial biopsy depends on the location of the lesion. Hence, there is substantial uncertainty in $\mathrm{E}$, but not in $\mathrm{P}_{\mathrm{KA}}$. Therefore, any comparisons between studies should be based on $\mathrm{P}_{\mathrm{KA}}$ and not on $\mathrm{E}$. $\mathrm{E}$ has been reported here to provide the reader a sense of scale of the radiation doses resulting from CBCTguided bronchoscopy. Data on radiation dose secondary to CBCT-guided bronchoscopy is extremely scant and reported as E. Bowling and coworkers reported a mean $\mathrm{E}$ of 4.3 (range, 3-5) $\mathrm{mSv}$ in a retrospective case series of 14 patients undergoing electromagnetic navigational bronchoscopy in combination with CBCT for peripheral lung, but there is no mention regarding their calculation method (12). A phantom study of CBCT and bronchoscopic navigation to a peripheral nodule by Hohenforst-Schmidt and coworkers reported very low body radiation doses of $0.98-1.15 \mathrm{mSv}$ from a single CBCT (17). Steinfort and coworkers recorded exposure parameters during 45 consecutive ultrasound bronchoscopies with fluoroscopic guidance with a mobile C-arm fluoroscopy system (18). The patient effective radiation dose was calculated with Monte Carlo computer simulations. The fluoroscopy time was short (96 \pm 55 seconds) in comparison to our study and hence the radiation exposure due to fluoroscopy was minimal (median effective radiation dose of $0.49 \pm 0.37$; range, $0.16-1.3 \mathrm{mSv}$ ). Understanding the limitations of conversion factors when reporting $\mathrm{E}$, the radiation dose described in our study seems acceptable when compared with other chest and abdomen diagnostic images and procedures (19-21).

The use of CBCT in combination with peripheral bronchoscopy has been briefly described in the literature (11-14). Hohenforst-Schmidt and coworkers combined CBCT with conventional bronchoscopy in a prospective feasibility study (11). A unique characteristic of their study was the creation of a virtual bronchoscopy pathway based on images obtained with CBCT, and the projection of this pathway over fluoroscopy images during bronchoscopy. RPEBUS was not utilized, and samples were only taken with biopsy forceps. A total of 33 lung patients were included in the study, and the overall diagnostic yield was $70 \%$. Twenty-three of their patients had lesions between 1-3 cm, and 10 had lesions greater than $3 \mathrm{~cm}$ in diameter. They reported 2 pneumothoraxes, but there was no mention on radiation dose. Park and coworkers retrospectively described their experience on 59 patients who underwent conventional bronchoscopy in combination with CBCT for peripheral lung lesions (13). Their target size was $3.1 \pm 1.0 \mathrm{~cm}$ (mean and SD). They described a diagnostic yield of $71.2 \%$, and they reported that confirmation of biopsy forceps within the lesion by CBCT was the most important factor associated with diagnostic yield. Radiation dose was not reported. Bowling and coworkers described a small case series with the combination of electromagnetic navigation, a transbronchial access tool, and CBCT for patients with peripheral lung lesions and no apparent "bronchus sign" on CT (12). They report an overall diagnostic yield of $71 \%$ (10/14 lesions), with only 3 of these 10 diagnoses being malignant in origin and 7 showing 
different inflammatory processes, mostly non-specific (12). Their only complication was one pneumothorax. Except for a case report by $\mathrm{Ng}$ and coworkers, we have not been able to find any other manuscripts on CBCT-guided bronchoscopy (14). Unlike the studies above, in our prospective study, in addition to documenting precisely radiation dose, we aimed to describe the potential additional yield provided by the use of CBCT during bronchoscopy. Our pre-CBCT diagnostic yield of 50\% (thin/ultrathin bronchoscopy and RP-EBUS) was slightly lower-but still within range - of what has been previously reported $(2,4,22)$. And our post-CBCT diagnostic yield of $70 \%$ is comparable to that of most techniques of guided-bronchoscopy (i.e., electromagnetic and non-electromagnetic navigation) (2). Interestingly, the four additional diagnoses achieved postCBCT were made in smaller targets. Factors that may have negatively impacted our diagnostic yield were the lack of bronchus sign (40\%), eccentric RP-EBUS image (55\%), the semisolid or ground-glass density of our lesions (40\%), and the presence of atelectasis obscuring the target lesion (20\%).

An interesting incidental finding of our study, and, to the best of our knowledge, never reported before, was the development of atelectasis in dependent areas during bronchoscopy. The atelectasis was not seen by fluoroscopy and was only detectable by CBCT so it would likely be missed in a standard bronchoscopy suite. This finding may be quite relevant, since atelectasis may mimic a solid lesion with RP-EBUS and can easily mislead an inexperienced bronchoscopist. And even if recognized by the bronchoscopist, it can still obscure the target if the latter is surrounded by atelectatic parenchyma. Both scenarios can negatively impact the diagnostic yield of bronchoscopy. All of our cases were performed under general anesthesia, which is likely the reason for the atelectasis. In addition, our procedures were relatively long due to a combination of factors: on-site cytology turn-around time, patient preparation for CBCT run (arms up positioning), CBCT review, and concomitant EBUS for staging in some cases. Atelectasis has been described in up to $90 \%$ of anesthetized patients undergoing different surgical procedures independent of age, gender, or utilized anesthetics (23). We believe this incidental finding deserves to be further studied since it may have an impact on our decision making when evaluating patients for peripheral bronchoscopy who have lung nodules located in dependent areas.

The main limitation of our study is its small sample size. The study was designed as a pilot to evaluate radiation safety. We can only describe the increase in navigational and diagnostic yield and the development of intra-procedural atelectasis as interesting findings, without drawing any strong conclusions.

\section{Conclusions}

The results of our study suggest that CBCT-guided bronchoscopy is associated with acceptable patient radiation dose, and that the addition of CBCT may potentially increase both navigation and diagnostic yield of thin/ultrathin bronchoscopy for peripheral lung nodules. The above findings as well as the incidental identification of intra-procedural atelectasis need to be confirmed in larger prospective studies.

\section{Acknowledgements}

We thank Lee Taylor, Justin Hair, Mehrnoosh Amirian, Linda Scott, Rodney Greene, and Roland Villareal for their assistance with bronchoscopy. Without their invaluable assistance, this study would have not been possible. We also thank Minxing Chen for his statistical support. This work was supported in part by Siemens.

\section{Footnote}

Conflicts of Interest: Dr. Casal has received research grants from Spiration and Siemens, and he is a paid consultant for Olympus and Boston Scientific. Dr. Tam has received research grants from Guerbet and Angiodynamics and she is a paid consultant for Merit Medical, Galil Medical, Jounce Therapeutics, and AbbVie. The other authors have no conflicts of interest to declare.

Ethical Statement: The study was performed at the University of Texas MD Anderson Cancer Center and it was approved by its Institutional Review Board (protocol 2016-0466). All participants gave informed consent before taking part in the study.

\section{References}

1. Gould MK, Donington J, Lynch WR, et al. Evaluation of individuals with pulmonary nodules: when is it lung cancer? Diagnosis and management of lung cancer, 3rd ed: American College of Chest Physicians evidence-based clinical practice guidelines. Chest 2013;143:e93-120S.

2. Wang Memoli JS, Nietert PJ, Silvestri GA. Meta-Analysis of Guided Bronchoscopy for the Evaluation of the 
Pulmonary Nodule. Chest 2012;142:385-93.

3. Steinfort DP, Khor YH, Manser RL, et al. Radial probe endobronchial ultrasound for the diagnosis of peripheral lung cancer: systematic review and meta-analysis. Eur Respir J 2011;37:902-10.

4. Ost DE, Ernst A, Lei X, et al. Diagnostic Yield and Complications of Bronchoscopy for Peripheral Lung Lesions. Results of the AQuIRE Registry. Am J Respir Crit Care Med 2016;193:68-77.

5. Eberhardt R, Anantham D, Ernst A, et al. Multimodality bronchoscopic diagnosis of peripheral lung lesions: a randomized controlled trial. Am J Respir Crit Care Med 2007;176:36-41.

6. Ost D, Shah R, Anasco E, et al. A randomized trial of CT fluoroscopic-guided bronchoscopy vs conventional bronchoscopy in patients with suspected lung cancer. Chest 2008;134:507-13.

7. Tsushima K, Sone S, Hanaoka T, et al. Comparison of bronchoscopic diagnosis for peripheral pulmonary nodule under fluoroscopic guidance with CT guidance. Respir Med 2006;100:737-45.

8. Heyer CM, Kagel T, Lemburg SP, et al. Transbronchial biopsy guided by low-dose MDCT: a new approach for assessment of solitary pulmonary nodules. AJR Am J Roentgenol 2006;187:933-9.

9. Matsuno Y, Asano F, Shindoh J, et al. CT-guided Ultrathin Bronchoscopy: Bioptic Approach and Factors in Predicting Diagnosis. Intern Med 2011;50:2143-8.

10. Orth RC, Wallac MWe, Kuo MD. C-arm Cone-beam CT: General Principles and Technical Considerations for Use in Interventional Radiology. J Vasc Interv Radiol 2008;19:814-20.

11. Hohenforst-Schmidt W, Zarogoulidis P, et al. Cone Beam Computertomography (CBCT) in Interventional Chest Medicine - High Feasibility for Endobronchial Realtime Navigation. J Cancer 2014;5:231-41.

12. Bowling MR, Brown C, Anciano CJ. Feasibility and Safety of the Transbronchial Access Tool for Peripheral Pulmonary Nodule and Mass. Ann Thorac Surg 2017;104:443-9.

13. Park SC, Kim CJ, Han CH, et al. Factors associated with the diagnostic yield of computed tomography-guided

Cite this article as: Casal RF, Sarkiss M, Jones AK, Stewart J, Tam A, Grosu HB, Ost DE, Jimenez CA, Eapen GA. Cone beam computed tomography-guided thin/ultrathin bronchoscopy for diagnosis of peripheral lung nodules: a prospective pilot study. J Thorac Dis 2018;10(12):6950-6959. doi: $10.21037 /$ jtd.2018.11.21 transbronchial lung biopsy. Thoracic Cancer 2017;8:153-8.

14. Ng CS, Yu SC, Lau RW, et al. Hybrid DynaCT-guided electromagnetic navigational bronchoscopic biopsy. Eur J Cardiothorac Surg 2016;49:i87-8.

15. Choi JW, Park CM, Goo JM, et al. C-arm cone-beam CT-guided percutaneous transthoracic needle biopsy of small ( $\leq 20 \mathrm{~mm}$ ) lung nodules: diagnostic accuracy and complications in 161 patients. AJR Am J Roentgenol 2012;199:W322-30.

16. Choo JY, Park CM, Lee NK, et al. Percutaneous transthoracic needle biopsy of small $(\leq 1 \mathrm{~cm})$ lung nodules under C-arm cone-beam CT virtual navigation guidance. Eur Radiol 2013;23:712-9.

17. Hohenforst-Schmidt W, Banckwitz R, Zarogoulidis P, et al. Radiation Exposure of Patients by Cone Beam CT during Endobronchial Navigation - A Phantom Study. J Cancer 2014;5:192-202.

18. Steinfort DP, Einsiedel P, Irving LB. Radiation Dose to Patients and Clinicians During Fluoroscopically-Guided Biopsy of Peripheral Pulmonary Lesions. Respir Care 2010;55:1469-74.

19. Mayo JR, Aldrich J, Muller NL. Radiation Exposure at Chest CT: A Statement of the Fleischner Society. Radiology 2003;228:15-21.

20. Guberina N, Forsting M, Ringelstein A, et al. Radiation exposure during CT-guided biopsies: recent CT machines provide markedly lower doses. Eur Radiol 2018;28:3929-35.

21. Demb J, Chu P, Nelson T, et al. Optimizing Radiation Doses for Computed Tomography Across Institutions: Dose Auditing and Best Practices. JAMA Intern Med 2017;177:810-7.

22. Tanner N, Pastis N, Walker A, et al. Multicenter, Prospective, Randomized Trial of Bronchoscopy With Ultrathin Bronchoscope and Radial Endobronchial Ultrasound With Fluoroscopy vs Standard Bronchoscopy With Fluoroscopy for Biopsy of Pulmonary Lesions. Chest 2017;152:A1123.

23. Tusman G, Bohnn SH, Warner DO, et al. Atelectasis and perioperative pulmonary complications in high-risk patients. Curr Opin Anaesthesiol 2012;25:1-10. 